\title{
Abandono terapêutico é o principal fator de casos refratários
}

\author{
Dr. Fabiano de Abreu Agrela Rodrigues \\ deabreu.fabiano@gmail.com
}

\section{RESUMO}

Alguns pacientes procuram ajuda profissional para lidar com suas questões emocionais. Sentimentos e emoções negativas que provocam reações desconfortáveis, gerando desprazer. Porém no processo terapêutico, quando iniciado o relato da "queixa principal”, fica evidente que são enrijecidos, inflexíveis, com personalidade cristalizada. Querendo "mudar o outro para si”, mas não querendo mudar em si comportamentos que geram sofrimentos para o próprio e para com quem convive. A terapia é personalizada, feita sob medida para as suas problemáticas. Não é para mudar o outro, e sim para que o paciente se perceba, se observe, se veja, se ouça. E promova ações que tenham desdobramentos positivos em sua própria vida. Mas às vezes o novo, mesmo com a possibilidade de ser melhor, assusta, desestabiliza. A pessoa prefere conviver com a velha dor, do que desbravar novos horizontes. O abandono terapêutico é um dos fatores que colabora para esse quadro.

Palavras-chave: terapeuta; tratamento; refratários; terapia 


\title{
Therapeutic dropout is the main factor in refractory cases
}

\begin{abstract}
Some patients seek professional help to deal with their emotional issues. Negative feelings and emotions, which cause uncomfortable reactions, generating displeasure. However, in the therapeutic process, when the report of the "main complaint" starts, it is evident that they are stiff, inflexible, with a crystallized personality. Wanting to "change the other for you", but not wanting to change yourself, behaviors that generate suffering for you and those you live with. The therapy is personalized, tailored to your own consumption. It is not to change the other, but for the patient to perceive himself, to observe himself, to see himself, to hear himself. And promote actions that have positive consequences in your own life. But sometimes the new, even with the possibility of being better, scares, destabilizes. And the person prefers to live with the old pain, then to open new horizons. Therapeutic abandonment is one of the factors that contributes to this situation.
\end{abstract}

Keywords: therapist; treatment; refractories; therapy

Artículo recibido: 02 enero 2022 Aceptado para publicación: 28 enero 2022 Correspondencia: deabreu.fabiano@gmail.com

Conflictos de Interés: Ninguna que declarar 


\section{INTRODUÇÃO}

Se um cliente busca a terapia porque, ao longo do processo, resiste em mudar o comportamento que tanto o faz sofrer e até mesmo abandona o tratamento pode ser por conflitos com o terapeuta ou mesmo uma fuga das hipóteses apresentadas pelo profissional. A resistência à mudança que melhoraria sua condição em relação ao meio em que vive e às pessoas com quem convive pode acontecer por falta de ajustamento num grau significativo para ele próprio ou para as pessoas relevantes do seu mundo. Mas no processo terapêutico a relação terapeuta-cliente é extremamente sensível.

O cliente não é uma fonte de dificuldades e o terapeuta por si só não é responsável pelo desenrolar da terapia. A qualidade da relação é proporcional à compreensão que terapeuta tem do seu cliente, bem como à sua habilidade para lidar com os problemas trazidos pelo paciente. Assim, o vínculo se aprofunda, e o cliente colhe os benefícios da atuação do terapeuta. Devido a inúmeras circunstâncias e variáveis, a terapia pode não alcançar os resultados almejados e esses casos são designados como refratários; problemas de difícil solução, tais como: pânico com agorafobia, respostas limitadas ao tratamento, ansiedade social, comportamento obsessivo-compulsivo, ansiedade generalizada, estresse pós-traumático, distúrbios alimentares, depressão, uso de substâncias, controle de impulso, problemas sexuais, insônia, cólera, hipocondria e ansiedade em relação à saúde, personalidade limítrofe, paralisias, comportamento antissocial e psicopatológico.

Entre as variáveis do cliente que não levam a ter êxito no tratamento destacamse: gerenciamento cognitivo ou intelectual baixo, conhecimento do problema pelo paciente, comorbidade e diagnóstico. É necessário ainda ter consciencia de que fatores associados ao terapeuta podem influir. Faz um sumário contrastivo do tratamento com base empírica e a prática com apoio empírico e salienta que a análise das falhas pode ser um bom aprendizado no desenvolvimento de intervenções mais eficazes e no refinamento de práticas empiricamente sustentadas.

\section{RESISTENTES AO TRATAMENTO}

Mesmo com o arsenal terapêutico disponível na atualidade muitos pacientes não apresentam resposta adequada, mostrando-se resistentes e até mesmo refratários aos tratamentos convencionais, o que contribui para a cronicidade dos diversos transtornos mentais. Exemplos de casos refratários são: esquizofrenia, transtornos do humor, 
transtornos de ansiedade, transtorno de estresse pós-traumático e transtorno obsessivocompulsivo. Thase (1999) aponta que as possíveis consequências da não remissão dos pacientes são: aumento das chances de recaídas em três vezes; prejuízos sociais e pessoais contínuos; maior utilização dos serviços de saúde, com aumento dos custos sociais; crescimento contínuo do risco de suicídio, do uso de substâncias psicoativas e da violência; e piora do prognóstico de outras condições médicas clínicas associadas. Paykel (1995) cita como exemplo o fato de pacientes com sintomas depressivos residuais apresentarem taxas de recaída próximas a $75 \%$, enquanto pacientes sem sintomas residuais atingem taxas de recaída de até $25 \%$. Não basta apenas aos terapeutas trabalhar para reduzir a intensidade dos sintomas. É importante que busquem alternativas para que se atinja a remissão dos transtornos, objetivando a rápida melhora do sofrimento, retomada do funcionamentopsicossocial e prevenção de episódios futuros, por exemplos. Fundamental nesse processo é que os profissionais reconheçam os fatores de risco para insuficiência ou ausência de resposta aos tratamentos psiquiátricos convencionais e a identificação de alternativas terapêuticas. Nesse contexto, cabe ressaltar a importância dos estudos sobre o processo terapêutico para a clínica psicológica. Castro (1999) corrobora essa questão destacando que esses estudos permitem a compreensão dos elementos envolvidos nas diferentes intervenções psicoterápicas, bem como o desenvolvimento e validação de estratégias, visando aprimorar o atendimento à saúde mental dos indivíduos. E, ainda, são essenciais as pesquisas que apontam caminhos para o avanço e melhor qualidade dos atendimentos psicológicos oferecidos.

\section{ABANDONO TERAPÊUTICO}

O abandono precoce do tratamento é uma das principais situações associadas à relação terapeuta e cliente. Bueno (1999) afirma que em relação às políticas públicas de saúde, o abandono ou interrupção do processo terapêutico é apontado como uma situação com implicações sérias nas trajetórias de saúde dos indivíduos e com alto custo econômico e social. Assim, a identificação das situações potencialmente associadas a um maior risco de abandono permite o desenvolvimento de ações preventivas tanto para o paciente como para o treinamento do profissional, orientando as ações dos programas em saúde mental. Cabe reforçar que o abandono em psicoterapia refere-se basicamente àquelas situações de interrupção do tratamento sem que haja indicação para tal encaminhamento 
por parte do terapeuta (Lhullier; Nunes, 2004). Goldenberg (2002) salienta em um de seus estudos que muitos clientes não retornam para o atendimento ou abandonam prematuramente a psicoterapia, sendo que a maioria dos casos de desistência acaba solicitando retorno de atendimento, necessitando reiniciar o processo diagnóstico e encaminhamento. No geral, o processo de retorno resulta no agravamento dos casos, na insatisfação profissional e em maior gasto econômico.

Walitzer; Dermen; Connors, (1999) aponta algumas características de personalidade associadas ao maior abandono terapêutico tais como isolamento social, agressividade, traços psicopáticos e baixa motivação. Em termos clínicos, os transtornos de personalidade em geral, os estados psicóticos e a ocorrência de ideação paranóide são os quadros com maior dificuldade de engajamento no processo terapêtico e os que apresentam mais risco de abandono (Bueno, 2001). Neste sentido, Romaro (2002) aponta que os transtornos de personalidade borderline são os casos de maior frequência de interrupção de tratamento em função das características transferenciais de hostilidade e dificuldade de vinculação. Hummelen, Wilberg e Karterud (2007) identificaram que, em situação de psicoterapia grupal, mulheres com diagnóstico de transtorno borderline não permanecem em atendimento, necessitando de um ambiente de maior contingência afetiva, visto a intensidade das emoções vividas no setting terapêutico. Outro fator associado à interrupção do tratamento foi destacado por Figueiredo; Schvinger (1981), ou seja, a falta de informação dos pacientes quanto ao processo terapêutico no início do atendimento, o que indica que muitos abandonos ocorrem pelo fato dos terapeutas não esclarecerem ao seu paciente como funciona a terapia.

Para Lhullier (2002) a descontinuidade de um tratamento está mais relacionada ao tipo de interação terapeuta-paciente do que com a técnica utilizada, destacando que a qualidade da aliança estabelecida é um fator responsável pelo sucesso de uma psicoterapia, mas ainda são poucos os estudos que investigam o abandono terapêutico em relação às diferentes modalidades terapêuticas. Vale ressaltar para um eficiente planejamento no campo clínico psicológico, que possibilite a identificação de falhas no tratamento, auxiliando na identificação de casos em risco de abandono e na prevenção de futuras desistências, é fundamental o conhecimento dos motivos. Estudar e identificar os fatores associados ao abandono e na prevenção de futuras desistências, e na redução dos casos refratários são de suma importância para delineamento do processo 
terapêutico. A eficácia terapêutica depende de uma atenção específica no preparo profissional e no planejamento dos serviços de saúde em relação ao desenvolvimento de intervenções voltadas para os grupos vulneráveis. Portanto, são necessárias açõesque possam auxiliar a redução desta alta incidência de abandono de tratamento por meio de abordagem e ações especiais, implementadas enquanto políticas públicas.

\section{CONSIDERAÇÕES FINAIS}

O abandono terapêutico se refere às situações de interrupção do processo terapêutico que estão associadas a diversos fatores, como características do paciente, do terapeuta, da técnica e do setting de trabalho, incluindo atendimentos realizados na clínica privada como em serviços de saúde comunitária. As situações associadas ao abandono psicoterápico constituem-se como importantes fontes de informação sobre os processos terapêuticos e possibilitam maior compreensão dos fatores envolvidos na eficácia dos atendimentos. É relevante para a prática clínica o desenvolvimento de ações específicas de pesquisa e de protocolos de atendimento voltados para a prevenção da interrupção precoce das psicoterapias.

\section{REFERÊNCIAS}

McKay, D., Taylor, S. \& Abramwitz, J. S. (Orgs.). (2011). Cognitive Behavioral Therapy and refratory cases: Turning failure into success. Washington, DC, US: American Psychological Association, xii+432 p. 1. Thase ME. Summary: defining remission in patients treated with antidepressants J Clin Psychiatry. 1999;60(Suppl 22):356. 3. Paykel ES, Ramana R, Cooper Z, Hayhurst H, Kerr J, Barocka A. Residual symptoms after partial remission: an important outcome in depression. Psychol Med. 1995;25(6):1171-80. Rev Bras Psiquiatr. 2007;29(Supl II):S39-40

CASTRO, P. F. Reflexões em psicologia e ciência: uma análise da pesquisa aplicada à Psicologia Clínica. Psicologia: Teoria e Prática,v. 1, n. 1, p. 3-13, 1999

BUENO, H. A.; CORDOBA, J. A.; ESCOLAR, P. A.; CARMONA, C. A.; RODRIGUEZ, G. C. et al. El abandono terapéutico. Actas Espain Psiquiatría, v. 29, n. 1, p. 33-40, 2001.

LHULLIER, A. C. Abandono de tratamento em psicoterapias realizadas numa clínicaescola. 2002. 183p. Tese (Doutorado em Psicologia) - Faculdade de Psicologia, Pontifícia Universidade Católica do Rio Grande do Sul, Porto Alegre, Brasil, 2002. 
GOLDENBERG, V. Ranking the correlates of psychotherapy duration. Administration and Policy in Mental Health, v. 29, n. 3, p. 201-214, 2002.

WALITZER, K. S.; DERMEN, K. H.; CONNORS, G. J. Strategies for preparing clients for treatment. A review. Behavior Modification, v. 23, n. 1, p. 29-51, 1999.

HUMMELEN, B.; WILBERG, T.; KARTERUD, S. Interviews of female patients with borderline personality disorder who dropped out of group psychotherapy. International Journal of Group Psychotherapy, v. 57, n. 1, p. 67-91, 2007.

ROMARO, R. A. O sentimento de exclusão social em personalidade borderline e o manejo da contratransferência. Mudanças, v. 10, n. 1, p. 65-71, 2002.

FIGUEIREDO, M. C. E.; SCHVINGER, A. A. Estratégias de atendimento psicológicoinstitucional a uma população carente. Arquivos Brasileiros de Psicologia, v. 33, n. 1, p. 46-57, 1981.

LHULLIER, A. C. Abandono de tratamento em psicoterapias realizadas numa clínicaescola. 2002. 183p. Tese (Doutorado em Psicologia) - Faculdade de Psicologia, Pontifícia Universidade Católica do Rio Grande do Sul, Porto Alegre, Brasil, 2002. 